\title{
GENERALIZED SASAKIAN-SPACE-FORMS WITH D-CONFORMAL CURVATURE TENSOR
}

\author{
Riddhi Jung Shah \\ Department of Mathematics \& Astronomy, Lucknow University, Lucknow-226007 \\ Corresponding address: shahrjgeo@ gmail.com \\ Received 17 January, 2012; Revised 21 August, 2012
}

\begin{abstract}
In this paper we study generalized Sasakian-space-forms with D-conformal curvature tensor. In generalized Sasakian-space-forms, we investigate some results on D-conformally flat, $\xi$-D-conformally flat, $\varphi$-Dconformally flat and the curvature condition $B(\xi, X) \cdot S=0$.
\end{abstract}

Mathematics Subject Classification 2010: 53C15, 53C25, 53D15.

Keywords: Sasakian-space-form, generalized Sasakian-space-form, D-conformal curvature tensor, $\eta$-Einstein manifold.

\section{INTRODUCTION}

A Sasakian manifold $M(\varphi, \xi, \eta, g)$ is said to be a Sasakian-space-form if all the $\varphi$-sectional curvatures $K(X \wedge \varphi X)$ are equal to a constant $c$, where $K(X \wedge \varphi X)$ denotes the sectional curvature of the section spanned by the unit vector field $X$, orthogonal to $\xi$ and $\varphi X$. In such a case, the Riemannian curvature tensor of $M$ is given by

$$
\begin{aligned}
R(X, Y) Z= & \frac{c+3}{4}\{g(Y, Z) X-g(X, Z) Y\} \\
+ & \frac{c-1}{4}\{g(X, \varphi Z) \varphi Y-g(Y, \varphi Z) \varphi X+2 g(X, \varphi Y) \varphi Z\} \\
+ & \frac{c-1}{4}\{\eta(X) \eta(Z) Y-\eta(Y) \eta(Z) X+g(X, Z) \eta(Y) \xi \\
& \quad-g(Y, Z) \eta(X) \xi\} .
\end{aligned}
$$

These spaces can be modeled, depending on $c>-3, c=-3$ or $c<-3$.

As a natural generalization of these manifolds Alegre, Blair and Carriazo introduced and studied the notion of generalized Sasakian-space-forms in 2004 [1]. They replaced constant quantities $(c+3) / 4$ and $(c-1) / 4$ of relation (1.1) by differentiable functions $f_{1}, f_{2}$ and $f_{3}$.

An almost contact metric manifold $M(\varphi, \xi, \eta, g)$ is said to be a generalized Sasakian-spaceform if the curvature tensor $R$ is given by [1] 


$$
\begin{aligned}
R(X, Y) Z & =f_{1}\{g(Y, Z) X-g(X, Z) Y\} \\
+ & f_{2}\{g(X, \varphi Z) \varphi Y-g(Y, \varphi Z) \varphi X+2 g(X, \varphi Y) \varphi Z\} \\
+ & f_{3}\{\eta(X) \eta(Z) Y-\eta(Y) \eta(Z) X+g(X, Z) \eta(Y) \xi \\
& \quad-g(Y, Z) \eta(X) \xi\}
\end{aligned}
$$

where $f_{1}, f_{2}, f_{3}$ are differentiable functions on $M$ and $X, Y, Z$ are vector fields on $M$. In such a case manifold is denoted by $M\left(f_{1}, f_{2}, f_{3}\right)$. In [2] authors studied contact metric and transSasakian generalized Sasakian-space-forms. In [5] and [6] authors studied on the locally $\varphi$ symmetric and $\eta$-recurrent Ricci tensor and on the projective curvature tensor respectively. Conformally flat generalized Sasakian-space-forms and locally symmetric generalized Sasakian-space-forms were studied by Kim [7]. Shukla and Shah studied on generalized Sasakian-space-forms with concircular curvature tensor [8].

A Riemannian manifold $M$ is said to be semi-symmetric if its curvature tensor $R$ satisfies [9]

$$
R(X, Y) \cdot R=0, \quad X, Y \in T M,
$$

where $R(X, Y)$ acts on $R$ as a derivation.

Generalized Sasakian-space-forms have also been studied by [10] and others.

\section{PRELIMINARIES}

In an almost contact metric manifold $M^{2 n+1}(\varphi, \xi, \eta, g)$, where $\varphi$ is a $(1,1)$ tensor field, $\xi$ is a contravariant vector field, $\eta$ is a 1 -form and $g$ is a compatible Riemannian metric, we have [3]

$$
\begin{aligned}
& \varphi^{2}(X)=-X+\eta(X) \xi, \eta(\xi)=1, \varphi \xi=0, \eta(\varphi X)=0 \\
& g(X, \xi)=\eta(X) \\
& g(\varphi X, \varphi Y)=g(X, Y)-\eta(X) \eta(Y) \\
& g(\varphi X, Y)=-g(X, \varphi Y) \\
& \left(\nabla_{X} \eta\right)(Y)=g\left(\nabla_{X} \xi, Y\right)
\end{aligned}
$$

In a $(2 n+1)$-dimensional generalized Sasakian-space-form the following relations hold:

$$
\begin{aligned}
& R(X, Y) \xi=\left(f_{1}-f_{3}\right)\{\eta(Y) X-\eta(X) Y\}, \\
& R(\xi, X) Y=\left(f_{1}-f_{3}\right)\{g(X, Y) \xi-\eta(Y) X\}, \\
& R(\xi, X) \xi=\left(f_{1}-f_{3}\right)\{\eta(X) \xi-X\},
\end{aligned}
$$




$$
\begin{aligned}
& S(X, Y)=\left(2 n f_{1}+3 f_{2}-f_{3}\right) g(X, Y)-\left(3 f_{2}+(2 n-1) f_{3}\right) \eta(X) \eta(Y), \\
& r=2 n(2 n+1) f_{1}+6 n f_{2}-4 n f_{3}, \\
& S(X, \xi)=2 n\left(f_{1}-f_{3}\right) \eta(X), \\
& \eta(R(X, Y) Z)=\left(f_{1}-f_{3}\right)\{g(Y, Z) \eta(X)-g(X, Z) \eta(Y)\} \\
& \eta(R(X, Y) \xi)=0 \\
& \eta(R(\xi, X) Y)=\left(f_{1}-f_{3}\right)\{g(X, Y)-\eta(X) \eta(Y)\} \\
& S(\varphi X, \varphi Y)=S(X, Y)-2 n\left(f_{1}-f_{3}\right) \eta(X) \eta(Y) .
\end{aligned}
$$

The D-conformal curvature tensor on a Riemannian manifold $\left(M^{2 n+1}, g\right)$ is defined as [4]

$$
\begin{aligned}
B(X, Y) Z & =R(X, Y) Z+\frac{1}{2(n-1)}[S(X, Z) Y-S(Y, Z) X \\
& +g(X, Z) Q Y-g(Y, Z) Q X-S(X, Z) \eta(Y) \xi \\
& +S(Y, Z) \eta(X) \xi-\eta(X) \eta(Z) Q Y+\eta(Y) \eta(Z) Q X] \\
& -\frac{k-2}{2(n-1)}[g(X, Z) Y-g(Y, Z) X] \\
+ & \frac{k}{2(n-1)}[g(X, Z) \eta(Y) \xi-g(Y, Z) \eta(X) \xi \\
& +\eta(X) \eta(Z) Y-\eta(Y) \eta(Z) X]
\end{aligned}
$$

where $k=\frac{r+4 n}{2 n-1}, R$ is the curvature tensor, $S$ is the Ricci tensor and $r$ is the scalar curvature.

\section{RESULTS AND DISCUSSION}

Definition: A $(2 n+1)$-dimensional generalized Sasakian-space-form $M\left(f_{1}, f_{2}, f_{3}\right)$ is said to be D-conformally flat if

$$
B(X, Y) Z=0 \text {. }
$$

Theorem 3.1. If a $(2 n+1)$-dimensional generalized Sasakian-space-form $M\left(f_{1}, f_{2}, f_{3}\right)$ is D-conformally flat, then $f_{3}=f_{1}+1$.

Proof. Let us consider a $(2 n+1)$-dimensional generalized Sasakian-space-form which satisfies the condition $B(X, Y) Z=0$, then from (2.16) we have 


$$
\begin{gathered}
0=R(X, Y) Z+\frac{1}{2(n-1)}[S(X, Z) Y-S(Y, Z) X+g(X, Z) Q Y \\
-g(Y, Z) Q X-S(X, Z) \eta(Y) \xi+S(Y, Z) \eta(X) \xi \\
-\eta(X) \eta(Z) Q Y+\eta(Y) \eta(Z) Q X] \\
-\frac{k-2}{2(n-1)}[g(X, Z) Y-g(Y, Z) X] \\
+\frac{k}{2(n-1)}[g(X, Z) \eta(Y) \xi-g(Y, Z) \eta(X) \xi \\
\quad+\eta(X) \eta(Z) Y-\eta(Y) \eta(Z) X] .
\end{gathered}
$$

Taking inner product on both sides of (3.2) by $W$, we get

$$
\begin{aligned}
0 & =R(X, Y, Z, W)+\frac{1}{2(n-1)}[S(X, Z) g(Y, W)-S(Y, Z) g(X, W) \\
& +S(Y, W) g(X, Z)-S(X, W) g(Y, Z)-S(X, Z) \eta(Y) \eta(W) \\
& +S(Y, Z) \eta(X) \eta(W)-S(Y, W) \eta(X) \eta(Z)+S(X, W) \eta(Y) \eta(Z)] \\
& -\frac{k-2}{2(n-1)}[g(X, Z) g(Y, W)-g(Y, Z) g(X, W)] \\
+ & \frac{k}{2(n-1)}[g(X, Z) \eta(Y) \eta(W)-g(Y, Z) \eta(X) \eta(W) \\
& +g(Y, W) \eta(X) \eta(Z)-g(X, W) \eta(Y) \eta(Z)]
\end{aligned}
$$

where $R(X, Y, Z, W)=g(R(X, Y) Z, W)$.

Setting $W=\xi$ in (3.3) and using (2.1) and (2.2), we obtain

$$
\begin{aligned}
0 & =\eta(R(X, Y) Z)+\frac{1}{2(n-1)}[S(Y, \xi) g(X, Z) \\
& -S(X, \xi) g(Y, Z)-S(Y, \xi) \eta(X) \eta(Z)+S(X, \xi) \eta(Y) \eta(Z) \\
& +2\{g(X, Z) \eta(Y)-g(Y, Z) \eta(X)\}] .
\end{aligned}
$$

Using (2.11) and (2.12) in (3.4), we get

$$
\left(\frac{f_{3}-f_{1}-1}{n-1}\right)\{g(Y, Z) \eta(X)-g(X, Z) \eta(Y)\}=0 .
$$

Since $g(Y, Z) \eta(X)-g(X, Z) \eta(Y) \neq 0$, we must have $f_{3}-f_{1}-1=0$, this implies that

$$
f_{3}=f_{1}+1 \text {. }
$$

This completes the proof of the theorem. 
Definition. Generalized Sasakian-space-form $M\left(f_{1}, f_{2}, f_{3}\right)$ of dimension $(2 n+1)$ is said to be $\xi$-D-conformally flat if

$$
B(X, Y) \xi=0
$$

Theorem 3.2. If a $(2 n+1)$-dimensional generalized Sasakian-space-form $M\left(f_{1}, f_{2}, f_{3}\right)$ satisfies the condition $B(X, Y) \xi=0$, then $f_{3}=f_{1}+1$.

Proof. Suppose the condition $B(X, Y) \xi=0$ holds in a $(2 n+1)$-dimensional generalized Sasakian-space-form. Then using (2.1) and (2.2) in (2.16), we have

$$
\begin{aligned}
0 & =R(X, Y) \xi+\frac{1}{2(n-1)}[S(X, \xi) Y-S(Y, \xi) X-S(X, \xi) \eta(Y) \xi \\
& +S(Y, \xi) \eta(X) \xi]+2[\eta(X) Y-\eta(Y) X] .
\end{aligned}
$$

In view of (2.6) and (2.11), (3.8) reduces to

$$
\left(\frac{f_{3}-f_{1}-1}{n-1}\right)\{\eta(Y) X-\eta(X) Y\}=0 .
$$

Since $\eta(Y) X-\eta(X) Y \neq 0$, we must have $f_{3}-f_{1}-1=0$, this implies that

$$
f_{3}=f_{1}+1 \text {. }
$$

Hence the theorem is proved.

From theorem 3.1 and theorem 3.2 we obtain the following:

Corollary 3.1. In a $(2 \mathrm{n}+1)$-dimensional generalized Sasakian-space-form $M\left(f_{1}, f_{2}, f_{3}\right)$ the curvature conditions $B(X, Y) Z=0$ and $B(X, Y) \xi=0$ are equivalent.

Definition. Let $M\left(f_{1}, f_{2}, f_{3}\right)$ be a $(2 n+1)$-dimensional generalized Sasakian-space-form. Then $M\left(f_{1}, f_{2}, f_{3}\right)$ is said to be $\varphi$-D-conformally flat if

$$
g(B(\varphi X, \varphi Y) \varphi Z, \varphi W)=0 .
$$

Theorem 3.3. If a $(2 n+1)$-dimensional generalized Sasakian-space-form is $\varphi$-Dconformally flat, then it is an $\eta$-Einstein manifold under the condition Tr. $\varphi=0$.

Proof. Let $M\left(f_{1}, f_{2}, f_{3}\right)$ be a $(2 n+1)$-dimensional generalized Sasakian-space-form. Suppose $M\left(f_{1}, f_{2}, f_{3}\right)$ satisfies the condition $g(B(\varphi X, \varphi Y) \varphi Z, \varphi W)=0$, then from (2.1) and (2.16), we have 


$$
\begin{aligned}
g(B(\varphi X, \varphi Y) \varphi Z, \varphi W) & =g(R(\varphi X, \varphi Y) \varphi Z, \varphi W) \\
& +\frac{1}{2(n-1)}[S(\varphi X, \varphi Z) g(\varphi Y, \varphi W)-S(\varphi Y, \varphi Z) g(\varphi X, \varphi W) \\
& +S(\varphi Y, \varphi W) g(\varphi X, \varphi Z)-S(\varphi X, \varphi W) g(\varphi Y, \varphi Z)] \\
& -\frac{k-2}{2(n-1)}[g(\varphi X, \varphi Z) g(\varphi Y, \varphi W)-g(\varphi Y, \varphi Z) g(\varphi X, \varphi W)] .
\end{aligned}
$$

In view of (3.11) and (3.12), we get

$$
\begin{aligned}
0 & =g(R(\varphi X, \varphi Y) \varphi Z, \varphi W)+\frac{1}{2(n-1)}[S(\varphi X, \varphi Z) g(\varphi Y, \varphi W) \\
& -S(\varphi Y, \varphi Z) g(\varphi X, \varphi W)+S(\varphi Y, \varphi W) g(\varphi X, \varphi Z) \\
& -S(\varphi X, \varphi W) g(\varphi Y, \varphi Z)] \\
& -\frac{k-2}{2(n-1)}[g(\varphi X, \varphi Z) g(\varphi Y, \varphi W) \\
& -g(\varphi Y, \varphi Z) g(\varphi X, \varphi W)] .
\end{aligned}
$$

By virtue of (1.2), (2.3), (2.4) and (2.15), (3.13) yields

$$
\begin{aligned}
0 & =f_{1}\{g(Y, Z) g(X, W)-g(Y, Z) \eta(X) \eta(W)-g(X, W) \eta(Y) \eta(Z) \\
& -g(X, Z) g(Y, W)+g(Y, W) \eta(X) \eta(Z)+g(X, Z) \eta(Y) \eta(W)\} \\
& +f_{2}\{g(X, \varphi Z) g(\varphi Y, W)-g(\varphi Y, Z) g(X, \varphi W) \\
& +2 g(X, \varphi Y) g(\varphi Z, W)\}+\frac{1}{2(n-1)}[S(X, Z) g(Y, W) \\
& -S(X, Z) \eta(Y) \eta(W)-2 n\left(f_{1}-f_{3}\right) g(Y, W) \eta(X) \eta(Z) \\
& -S(Y, Z) g(X, W)+S(Y, Z) \eta(X) \eta(W)+2 n\left(f_{1}-f_{3}\right) g(X, W) \eta(Y) \eta(Z) \\
& +S(Y, W) g(X, Z)-S(Y, W) \eta(X) \eta(Z)-2 n\left(f_{1}-f_{3}\right) g(X, Z) \eta(Y) \eta(W) \\
& \left.-S(X, W) g(Y, Z)+S(X, W) \eta(Y) \eta(Z)+2 n\left(f_{1}-f_{3}\right) g(Y, Z) \eta(X) \eta(W)\right] \\
& -\frac{k-2}{2(n-1)}[g(X, Z) g(Y, W)-g(X, Z) \eta(Y) \eta(W)-g(Y, W) \eta(X) \eta(Z) \\
& -g(Y, Z) g(X, W)+g(Y, Z) \eta(X) \eta(W)+g(X, W) \eta(Y) \eta(Z)] .
\end{aligned}
$$

Let $\left\{e_{i}: i=1,2, \ldots \ldots \ldots, 2 n+1\right\}$ be an orthonormal basis of the tangent space at any point of 
the manifold. Putting $X=W=e_{i}$ in (3.14) and taking summation over $i, 1 \leq i \leq 2 n+1$, we get

$$
\begin{aligned}
0 & =(2 n-1) f_{1}\{g(Y, Z)-\eta(Y) \eta(Z)\} \\
& +f_{2}\{3 g(\varphi Y, \varphi Z)-g(\varphi Y, Z) \operatorname{Tr} . \varphi\} \\
& +\frac{1}{2(n-1)}[-2(n-1) S(Y, Z)-S(Z, \xi) \eta(Y) \\
& +\left\{2 n(2 n-1)\left(f_{1}-f_{3}\right)+r\right\} \eta(Y) \eta(Z) \\
- & \left.S(Y, \xi) \eta(Z)+\left\{2 n\left(f_{1}-f_{3}\right)-r\right\} g(Y, Z)\right] \\
+ & \frac{r+2}{2(n-1)}[g(Y, Z)-\eta(Y) \eta(Z)] .
\end{aligned}
$$

By the use of (2.3) and (2.11), (3.15) reduces to

$$
\begin{aligned}
S(Y, Z) & =\left[\frac{\left(2 n^{2}-2 n+1\right) f_{1}+3(n-1) f_{2}-n f_{3}+1}{n-1}\right] g(Y, Z) \\
& +\left[\frac{n(3-2 n) f_{3}-f_{1}-3(n-1) f_{2}-1}{n-1}\right] \eta(Y) \eta(Z),
\end{aligned}
$$

under the condition $\operatorname{Tr} . \varphi=0$.

From (3.16) we get

$$
S(Y, Z)=\alpha g(Y, Z)+\beta \eta(Y) \eta(Z),
$$

where

$$
\begin{aligned}
& \alpha=\frac{\left(2 n^{2}-2 n+1\right) f_{1}+3(n-1) f_{2}-n f_{3}+1}{n-1} \text { and } \\
& \beta=\frac{n(3-2 n) f_{3}-f_{1}-3(n-1) f_{2}-1}{n-1} .
\end{aligned}
$$

The relation (3.17) implies that the manifold is an $\eta$-Einstein manifold. This completes the proof of the theorem.

Theorem 3.4. A ( $2 n+1)$-dimensional generalized Sasakian-space-form $M\left(f_{1}, f_{2}, f_{3}\right)$ satisfying the condition $B(\xi, X) . S=0$ is an Einstein manifold and has the scalar curvature $r=2 n(2 n+1)\left(f_{1}-f_{3}\right)$.

Proof. Let $M\left(f_{1}, f_{2}, f_{3}\right)$ be a $(2 n+1)$-dimensional generalized Sasakian-space-form. Suppose that $M\left(f_{1}, f_{2}, f_{3}\right)$ satisfies the condition $(B(\xi, X) . S)(U, V)=0$, where $S$ is the Ricci tensor. Then we have

$$
S(B(\xi, X) U, V)+S(U, B(\xi, X) V)=0 .
$$


In view of (2.1), (2.2), (2.7) and (2.11), (2.16) yields

$$
\begin{array}{r}
B(\xi, Y) Z=\left(f_{1}-f_{3}-\frac{1}{n-1}\right)\{g(Y, Z) \xi-\eta(Z) Y\} \\
+\frac{1}{2(n-1)}\left[2 n\left(f_{1}-f_{3}\right)\{Y-\eta(Y) \xi\} \eta(Z)\right. \\
-\{g(Y, Z)-\eta(Y) \eta(Z)\} Q \xi] .
\end{array}
$$

Using (3.19) in (3.18) we get

$$
\begin{aligned}
0 & =\left(f_{1}-f_{3}-\frac{1}{n-1}\right)\left\{2 n\left(f_{1}-f_{3}\right) g(X, U) \eta(V)-S(X, V) \eta(U)\right\} \\
& +\frac{n\left(f_{1}-f_{3}\right)}{n-1}\left[\left\{S(X, V)-2 n\left(f_{1}-f_{3}\right) \eta(X) \eta(V)\right\} \eta(U)\right. \\
& \left.-2 n\left(f_{1}-f_{3}\right)\{g(X, U)-\eta(X) \eta(U)\} \eta(V)\right] \\
& +\left(f_{1}-f_{3}-\frac{1}{n-1}\right)\left\{2 n\left(f_{1}-f_{3}\right) g(X, V) \eta(U)-S(X, U) \eta(V)\right\} \\
& +\frac{n\left(f_{1}-f_{3}\right)}{n-1}\left[\left\{S(X, U)-2 n\left(f_{1}-f_{3}\right) \eta(X) \eta(U)\right\} \eta(V)\right. \\
& \left.-2 n\left(f_{1}-f_{3}\right)\{g(X, V)-\eta(X) \eta(V)\} \eta(U)\right] .
\end{aligned}
$$

Putting $V=\xi$ in (3.20) and using (2.1), (2.2) and (2.11), we obtain

$$
S(X, U)=2 n\left(f_{1}-f_{3}\right) g(X, U) .
$$

The relation (3.21) implies that the generalized Sasakian-space-form is an Einstein manifold. Again, taking an orthonormal frame field at any point of the manifold and contracting over $X$ and $U$ in (3.21) we have

$$
r=2 n(2 n+1)\left(f_{1}-f_{3}\right),
$$

where $r$ is the scalar curvature.

In view of (3.21) and (3.22), the theorem is proved.

\section{REFERENCES}

[1] Alegre, P, Blair, D E \& Carriazo, A, Generalized Sasakian-space-form, Israel J. Math., 141(2004), 157.

[2] Alegre, P \& Carriazo, A, Structures on generalized Sasakian-space-forms, Differential Geom. Appl., 26(2008), 656.

[3] Blair, D E, Contact manifolds in Riemannian geometry, Lecture notes in Mathematics, 509(1976), Springer, Verlag, Berlin.

[4] Chuman, G, On the D-conformal curvature tensor, Tensor N. S. 46(1983), 125. 
[5] De, U C \& Sarkar, A, Some results on generalized Sasakian-space-forms, Thai J. of Math., 8(2010), No. 1, 1.

[6] De, U C \& Sarkar, A, On the projective curvature tensor of generalized Sasakianspace-forms, Quaestiones Mathematicae, 33(2010), No. 2, 245.

[7] Kim, U K, Conformally flat generalized Sasakian-space-forms and locally symmetric generalized Sasakian-space-forms, Note di Matematica, 26(2006), No.1, 55.

[8] Shukla, N V C \& Shah R J, Generalized Sasakian-space-forms with Concircular curvature tensor, J. Rajasthan Acad. Phy. Sci., 10(2011), No. 1, 11.

[9] Szabo, Z I, Structure theorems on Riemannian spaces satisfying $R(X, Y) \cdot R=0, \mathrm{I}$. The local version, J. Diff. Geom., 17(1982), 531.

[10] Yadav, S, Suthar, D L \& Shrivastava, A K, Some results on $M\left(f_{1}, f_{2}, f_{3}\right)_{2 n+1}$ manifolds, International J. of Pure and Appl. Math. 70(2011), 415. 